\title{
14q12 and severe Rett-like phenotypes: new clinical insights and physical mapping of FOXG1-regulatory elements
}

\author{
Lila Allou ${ }^{1,15}$, Laetitia Lambert ${ }^{1,2,3,15}$, Daniel Amsallem ${ }^{4}$, Eric Bieth ${ }^{5}$, Patrick Edery ${ }^{6,7}$, Anne Destrée ${ }^{8}$,
} François Rivier ${ }^{9}$, David Amor ${ }^{10}$, Elizabeth Thompson ${ }^{11}$, Julian Nicholl ${ }^{12}$, Michael Harbord ${ }^{13}$, Christophe Nemos ${ }^{1}$, Aline Saunier ${ }^{14}$, Aissa Moustaïne ${ }^{14}$, Adeline Vigouroux ${ }^{5}$, Philippe Jonveaux ${ }^{1,14}$ and Christophe Philippe ${ }^{\star, 1,14}$

The Forkhead box G1 (FOXG1) gene has been implicated in severe Rett-like phenotypes. It encodes the Forkhead box protein G1, a winged-helix transcriptional repressor critical for forebrain development. Recently, the core FOXG1 syndrome was defined as postnatal microcephaly, severe mental retardation, absent language, dyskinesia, and dysgenesis of the corpus callosum. We present seven additional patients with a severe Rett-like neurodevelopment disorder associated with de novo FOXG1 point mutations (two cases) or 14q12 deletions (five cases). We expand the mutational spectrum in patients with FOXG1-related encephalopathies and precise the core FOXG1 syndrome phenotype. Dysgenesis of the corpus callosum and dyskinesia are not always present in FOXG1-mutated patients. We believe that the FOXG1 gene should be considered in severely mentally retarded patients (no speech-language) with severe acquired microcephaly ( -4 to $-6 \mathrm{SD}$ ) and few clinical features suggestive of Rett syndrome. Interestingly enough, three $14 \mathrm{q} 12$ deletions that do not include the FOXG1 gene are associated with phenotypes very reminiscent to that of FOXG1-mutation-positive patients. We physically mapped a putative long-range FOXG1-regulatory element in a $0.43 \mathrm{Mb}$ DNA segment encompassing the PRKD1 locus. In fibroblast cells, a cis-acting regulatory sequence located more than $0.6 \mathrm{Mb}$ away from FOXG1 acts as a silencer at the transcriptional level. These data are important for clinicians and for molecular biologists involved in the management of patients with severe encephalopathies compatible with a FOXG1-related phenotype.

European Journal of Human Genetics (2012) 20, 1216-1223; doi:10.1038/ejhg.2012.127; published online 27 June 2012

Keywords: 14q12; CNV; FOXG1; Rett syndrome; cis-acting regulatory element

\section{INTRODUCTION}

Point mutations in the Forkhead box G1 (FOXG1) gene, encoding a brain-specific transcriptional repressor essential for the development of the telencephalon, were found to be responsible for congenital $^{1-7}$ or classical $^{2,5}$ Rett syndrome (RTT) in females and also in males. ${ }^{5-7}$ Copy number variations (CNV) in 14q12 containing the FOXG1 gene have been identified in both genders in patients suffering from neurodevelopmental disorders. Microdeletions in $14 \mathrm{q} 12$ were described in patients with early-onset Rett-like phenotypes associated with facial dysmorphic features. ${ }^{8}$ In one case, a selective FOXG1 deletion resulted in congenital Rett variant with minor facial dysmorphism (synophrys and pointed chin). ${ }^{9} \quad 14 \mathrm{q} 12$ microduplications encompassing the FOXG1 locus also result in abnormal neurodevelopmental phenotype consisting of epilepsy (especially infantile spasms), mental retardation, and severe speech impairment. ${ }^{10-13}$ Although the $14 \mathrm{q} 12 \mathrm{CNV}$ s contain additional genes, abnormal dosage of FOXG1 is considered to be the best candidate to explain the neurodevelopmental disorders in these patients. Recently, Kortüm et $a l^{6}$ reported an extensive clinical evaluation in a large series of patients heterozygous for a deleterious allele in FOXG1. The authors reported both point mutations and CNVs in 14q12, and proposed the designation of FOXG1 syndrome for the clinical phenotype in patients with a FOXG1 mutation. Patients with a chromosomal breakpoint ${ }^{6,14}$ or deletions in $14 \mathrm{q}^{6,6,8}$ close but not disrupting the FOXG1 gene have a phenotype similar to that of FOXG1-positive individuals. A position effect that causes altered expression of FOXG1 has been proposed to explain the clinical features in these individuals. ${ }^{6,14}$

${ }^{1}$ EA 4368, Déficiences mentales et anomalies de structure du génome, Faculté de Médecine, Université de Lorraine, Vandoeuvre-les-Nancy, France; ${ }^{2}$ Service de Médecine Infantile 1, Hôpital d'Enfants, Centre Hospitalier Régional et Universitaire, Rue du Morvan, Vandoeuvre-les-Nancy, France; ${ }^{3}$ Unité de Génétique Clinique, Médecine Néonatale, Maternité Régionale Universitaire, Rue du Dr Heydenreich, Nancy, France; ${ }^{4}$ Service de Neuropédiatrie, Hôpital St Jacques, Place St Jacques, Besançon, France; 5 Service de Génétique Médicale, Hôpital Purpan, Place du Dr Baylac, Toulouse, France; ${ }^{6}$ Service de Cytogénétique Constitutionnelle, Hospices civils de Lyon, Groupement Hospitalier Est,

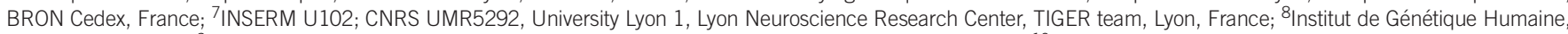
Charleroi, Belgique; ${ }^{9} \mathrm{CHRU}$ Montpellier, Neuropédiatrie CR Maladies Neuromusculaires, Montpellier, France; ${ }^{10}$ Murdoch Children's Research Institute, Royal Children's Hospital, Melbourne, Australia; ${ }^{11}$ SA Clinical Genetics Service, SA Pathology, Women's and Children's Hospital, North Adelaide, SA, Australia; ${ }^{12}$ Department of Cytogenetics, SA Pathology, Women's and Children's Hospital, North Adelaide, South Australia; ${ }^{13}$ Department of Paediatric, Flinders Medical Centre, Bedford Park, South Australia; ${ }^{14}$ Laboratoire de génétique médicale, Centre Hospitalier Régional et Universitaire, Rue du Morvan, Vandoeuvre-les-Nancy, France

15These authors contributed equally to this work.

*Correspondence: Dr C Philippe, EA 4368 UHP Nancy Universite, Laboratoire de génétique médicale, Centre Hospitalier Universitaire de Nancy Brabois, Rue du Morvan, Vandoeuvre les Nancy F-54511, France. Tel: +33 3831576 04; Fax: +33 3831537 72; E-mail: c.philippe@chu-nancy.fr

Received 31 August 2011; revised 3 April 2012; accepted 24 April 2012; published online 27 June 2012 
Here, we described seven new deleterious alleles $14 \mathrm{q} 12$ consisting of two point mutations in FOXG1, three CNVs encompassing FOXG1, and two deletions distal but close to the FOXG1 locus. The patients (four girls and three boys) presented with severe RTT-like neurodevelopment disorders. Using cultured fibroblasts, we determined the impact of three $14 \mathrm{q} 12$ CNVs on the expression of FOXG1. We confirmed and precisely mapped cis-acting regulatory elements distal to FOXG1 that may act at the transcriptional level.

\section{PATIENTS AND METHODS}

\section{Patients and phenotype definitions}

In this study, we included individuals with Rett and severe Rett-like phenotypes who were recruited at different clinical genetic centres through France, Australia, and Belgium. The molecular and cytogenetic studies were performed at the molecular laboratory of the public hospital in Nancy, France. We tested typical RTT and congenital or early-onset RTT when the normal perinatal period was absent or shorter than 6 months. For almost all patients, mutations in MECP2 and CDKL5 genes could not be detected in these patients. We also included patients with a severe mental retardation associated with epilepsy, these individuals were negative for $M E F 2 C$. Total genomic DNA was extracted from peripheral blood using the Nucleon BACC genomic DNA extraction kit (GE Healthcare, Velizy-Villa Coubley, France). Biological samples from patients and clinical data were obtained after informed consent at all participating institutions.

\section{Mutation screening of FOXG1 by direct sequencing of PCR products}

We analysed the entire coding sequence by direct sequencing of PCR products. Exon 1 of the FOXG1 gene was PCR amplified (primer sequences available upon request). Primers were modified by the addition of either M13F $\left(5^{\prime}\right.$-tgtaaaacgacggccagt- $\left.3^{\prime}\right)$ or M13R ( $5^{\prime}$-caggaaacagtcatgacc- $\left.3^{\prime}\right)$ sequences at their $5^{\prime}$-end. The coding sequence was screened by direct DNA sequencing with M13F and M13R primers as described earlier. ${ }^{15}$ Sequences were automatically analysed with the Seqscape 2.5 software (Applied Biosystems, Foster City, CA, USA). Sequence variants are numbered starting from the first base of the ATG codon, numbering based on reference sequence NM_005249.3. Naming of variants with the Alamut 2.02 software (Interactive Biosoftware, Courtaboeuf, France) follows the Human Genome Variation Society nomenclature.

\section{Screening for large rearrangements of FOXG1 by qPCR}

Detection of large rearrangements of the FOXG1 gene was performed by qPCR (quantitative PCR) for exon 1 (two amplicons) and exon 5 (one amplicon) (primer sequences available upon request). qPCR on genomic DNA was performed as described previously, ${ }^{16}$ using the ABI PRISM 7500 Sequence Detection System (Applied Biosystems).

\section{Array CGH}

The $14 \mathrm{q} 12$ deletion in patient 3 was identified by screening 18 patients with the Agilent kit 105A (Agilent Technologies, Santa Clara, CA, USA). This work was supported by a grant (CPRC 04.9582, 2009) from the Centre Hospitalier Universitaire de Nancy. The array-comparative genomic hybridisation (CGHa) analysis was performed as described previously. ${ }^{16}$ The array was analysed with an Agilent scanner and the Feature Extraction software (v10.7.3.1, Essone, Massy, France). A graphical overview was obtained using CGH analytics software (v4.0.76, Essone). Validation of CNVs identified by CGHa was performed by qPCR. For each CNV, we tested three primer sets located in the chromosomal region of interest to establish the de novo/inherited feature of the chromosomal imbalance.

\section{Cell culture}

Fibroblast cells were maintained in Dulbecco's Modified Eagle's Medium (Gibco-Invitrogen, Illkirch, France) supplemented with 10\% fetal bovine serum and antibiotics at $37^{\circ} \mathrm{C}, 5 \% \mathrm{CO}_{2}$ under humidified atmosphere.

\section{Analysis of the FOXG1 transcript by RT-qPCR}

Total RNAs from fibroblast cells (patients 3, 6, 7, and 8) were extracted with the TRIzol reagent (Invitrogen, Courtaboeuf, France). For patient 5, total RNAs were extracted from saliva with the Oragene-RNA Self-Collection Kit (DNA Genotek, Kanata, ON, Canada). RNA quality (RNA Integrity Number >9) was assessed with the Agilent 2100 Bioanalyzer. Before RT-PCR, RNAs were treated with DNase I (Sigma, Saint Quentin Fallavier, France) at room temperature for $15 \mathrm{~min}$, DNase I was inactivated at $70^{\circ} \mathrm{C}$ for $10 \mathrm{~min}$. RT-PCRs were performed with primers located in exon 1 of FOXG1 with the QIAGEN (Courtaboeuf, France) OneStep kit. Reverse transcriptase quantitative real-time PCR (RT-qPCR) was performed on an ABI PRISM 7500 Sequence Detection System (Applied Biosystems) as described previously. ${ }^{16}$ The dosage of the FOXG1-derived mRNAs was performed with one amplicon (primer sequences available upon request). The relative quantification was performed with the $2^{-D D C t}$ method relative to $E S D$ cDNAs as described previously. ${ }^{16}$ All samples were run in triplicate.

\section{Analysis of the FOXG1 transcript by western blot}

Proteins were isolated from fibroblast cells (patients 3, 6, 7, and 8) with the ProteoJET Cytoplasmic and Nuclear Protein Extraction kit (Frementas, ZA Courtaboeuf, France), and the concentration was determined using bovine serum albumin as a standard (BCA Protein assay, Pierce Biotechnology, Rockford, IL, USA). Total lysates were boiled in SDS sample buffer, separated by SDS-PAGE, and blotted to polyvinylidene fluoride membrane (Biorad, Marne la Coquette, France). Filters were blocked in tris-buffered saline tween $20(0.1 \%)$ (TBST) (10 mm Tris-HCl, pH 8.0, $150 \mathrm{~mm} \mathrm{NaCl}$, and 0.05\% tween 20) plus $5 \%$ dried milk, and incubated with primary antibodies for $16 \mathrm{~h}$ at $4{ }^{\circ} \mathrm{C}$. The following primary antibodies were used: rabbit polyclonal antiFOXG1 (1:1000, Abcam, Paris, France) and rabbit polyclonal anti-beta Actin (1:2000, Abcam). After washing three times with TBST, filters were incubated with peroxidase-conjugated secondary antibody (anti-rabbit IgG; 1:2000/ 1:5000; Abcam) for $1 \mathrm{~h}$ at room temperature. Detection was performed by enhanced chemiluminescence (ECL Plus, Amersham, GE Healthcare) using the ChemiDoc software (BioRad, Marnes-la-Coquette, France)

\section{Use of online bioinformatics tools}

For the identification of conserved non-coding elements, we used the ESPERR (evolutionary and sequence pattern extraction through reduced representations, http://genome.cse.ucsc.edu/) computational method and the DCODE ECR browser (http://ecrbrowser.dcode.org) with the human March 2006 (hg18) assembly. We selected highly conserved non coding elements (HCNE) with a vertical viewing range between (0.25-0.4 (ESPERR) or containing a minimum of 100 bases with at least 70\% identity (ECR browser)).

\section{Description of sequence variants}

Sequences variant in the FOXG1 gene are numbered starting from the first base of the ATG codon, numbering based on reference sequence NM_001453.2. For CNVs detected by CGHa, the distances from the $14 \mathrm{p}$ telomere are derived from the NCBI genome browser (www.ncbi.nlm.nih.gov/gap, build 36).

\section{RESULTS}

Identification of point mutations in FOXG1 and CNVs in $14 \mathrm{q} 12$ In the French laboratory, the molecular screening of FOXG1 was performed by sequencing analysis and qPCR in 80 patients with RTT or RTT-like encephalopathies. These patients were negative for MECP2 and CDKL5. For 18 patients with typical RTTs (nine cases), Hanefeld variants (five cases), or congenital RTTs (four cases), we also looked for CNVs by CGHa. Patients 1, 3, 6, and 7 were initially tested negative for MECP2. Both MECP2 and CDKL5 were excluded in patient 2. CNVs were identified by CGHa as a first-tier diagnostic test for patients 4,5 , and 8 . We identified two point mutations and two CNVs in 14q12 (patients 1, 2, 3, and 4, Figure 1a). The c.256dupC/c.256delC were identified in patients 1 and 2, respectively. These in/del mutations result in frameshifts (p.Gln86ProfsX35/ p.Gln86ArgfsX106), theoretically the resulting proteins miss all 
a

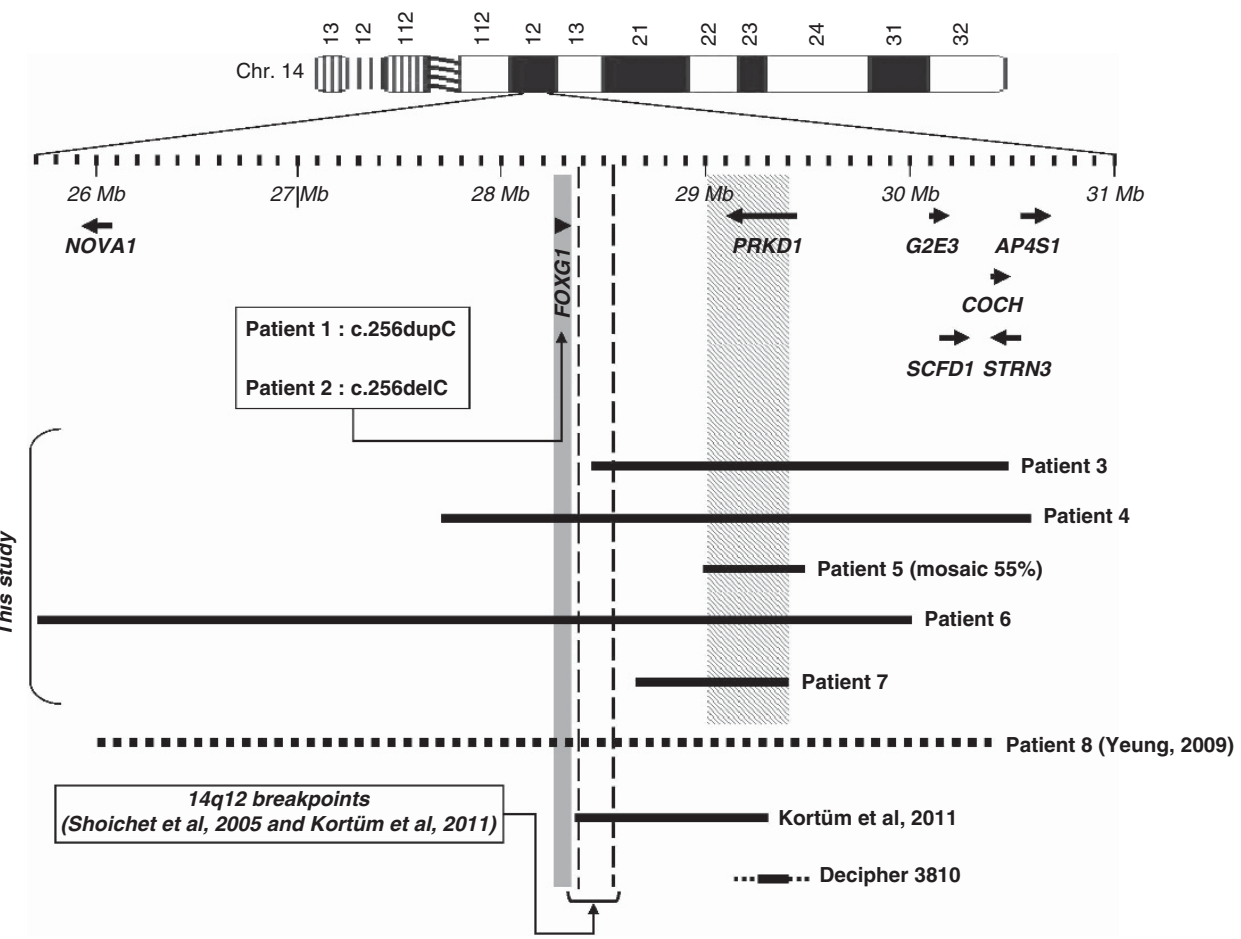

b Chr14: 28.945.423- 29.372.834

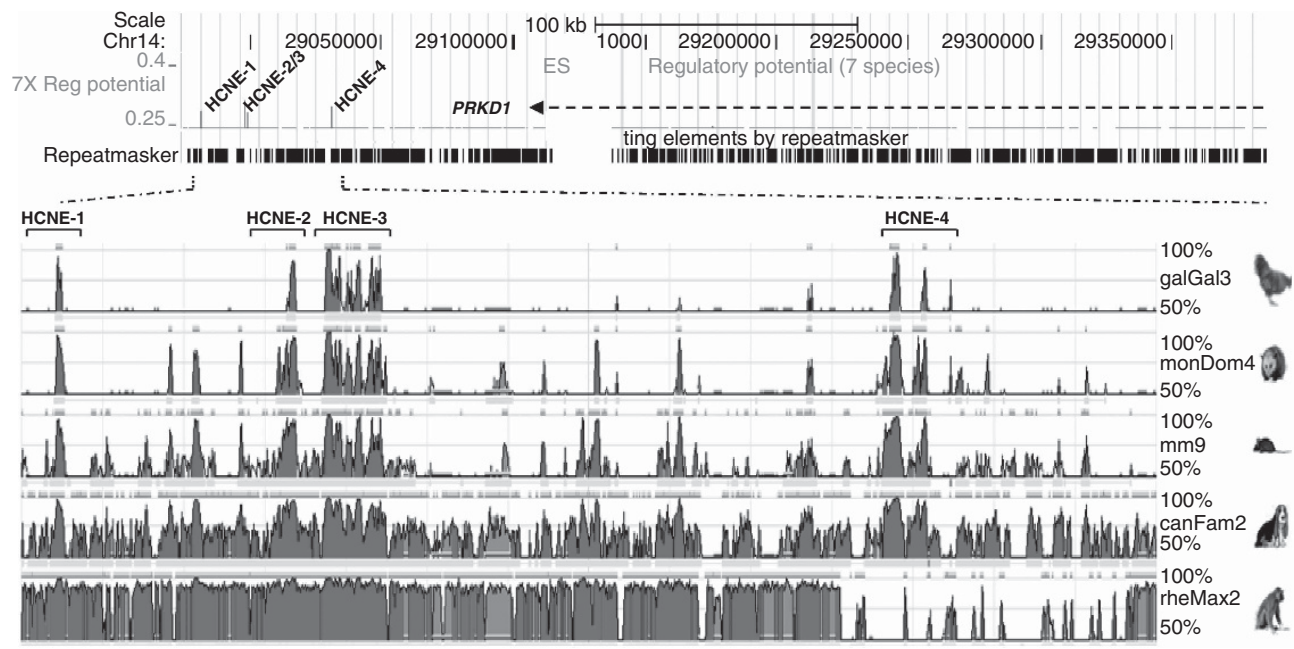

Figure 1 Physical mapping of FOXG1-regulatory elements in 14q12. (a) CNVs in 14q12 and FOXG1-related encephalopathies. We identified two point mutations in FOXG1 (patients 1 and 2) and five CNVs in 14q12 encompassing or close to FOXG1 (patients 3-7). The detailed view of the 14q12 region is derived from the NCBI genome browser (hg18, March 2006 assembly). Genes in 14q12 are represented by arrows $\left(5^{\prime} \rightarrow 3^{\prime}\right.$ orientation). Deletions are depicted by horizontal black lines and duplication is depicted by an horizontal black dashed line. Vertical dashed black lines depict the location of $14 q 12$ breakpoint in patients with a t(2;14). The minimal deleted region (28945423-29372 834) is materialised by diagonal lines. (b) Bioinformatic predictions of putative cis-regulatory elements for the FOXG1 gene. In the minimal deleted segment, the FOXG1/PRKD1 intergenic region contains four HCNEs (HCNE1: 28963 948-28 965 333; HCNE2: 28981 071-28 982 265; HCNE3: 29030 242-29 031 756; HCNE4: 29 066 922-29 068 642) identified by using the ESPRR computational method. The conservation throughout rhesus monkey, dog, mouse, opossum,and chicken of fragments $>100 \mathrm{bp}$ at $70 \%$ identity are indicated in red using the ECR browser (http://ecrbrowser.dcode.org).

functional domains of the FOXG1 transcription factor. The deletion in patient 3 is distal to the FOXG1 locus. It extends on $2.1 \mathrm{Mb}$ of DNA in $14 \mathrm{q} 12$ and is located $<100 \mathrm{~kb}$ to FOXG1. The deletion in patient 3 was identified by CGHa, it includes 5 RefSeq genes: 14 cenPRKD1-G2E3-SCFD1-COCH-STRN3-14qtel. Patient 4 is heterozygous for a $3.2 \mathrm{Mb}$ deletion that was identified by $\mathrm{qPCR}$ as part of targeted FOXG1 screening. This deletion encompasses FOXG1 as well as six other RefSeq genes (PRKD1-G2E3-SCFD1-COCH-STRN3$A P 4 S 1)$. CNVs in patients $5-8$ were identified by other laboratories. Patient 5 is a somatic mosaic for a $0.51 \mathrm{Mb}$ deletion in $14 \mathrm{q} 12$ that removes part of the PRKD1 (protein kinase D1) gene (exons 2-18). The neighbouring genes (FOXG1 and G2E3) are not affected by this small deletion. FISH analysis on lymphocyte nuclei with BAC clones RP11-566C18 and RP11-181I14 revealed a 55\% rate of mosaicism 
(data not shown). Patient 6 (DECIPHER 251718) carries a $4.1 \mathrm{Mb}$ deletion in 14q12 including NOVA1, FOXG1, and PRKD1. In patient 7 , a CGHa analysis revealed a $0.62 \mathrm{Mb}$ deletion distal to FOXG1, which encompasses part of the PRKD1 gene (exons 2-18). In all seven cases, testing of parents revealed that the mutation occurred de novo. Sequencing analysis of the FOXG1 coding region did not reveal any point mutation on the second allele in patients 3-7. Patient 8 was the first duplication in $14 \mathrm{q} 12$ including FOXG1 described in the literature. ${ }^{12}$ The microduplication extends on $4.45 \mathrm{Mb}$ of DNA and involves six RefSeq genes: 14cen-NOVA1FOXG1-PRKD1-G2E3-SCFD1-COCH-14qtel.

\section{Clinical reports}

Complete clinical descriptions for patients 1-7 are provided in the Supplementary Information. A summary is presented in Table 1. No common facial feature is remarkable in our cohort of seven FOXG1mutated patients (Figure 2).

\section{Quantification of the FOXG1 expression}

We established fibroblasts cultures from skin for patients 3, 6, and 7 . For patient 8 , we used fibroblasts from a cell line that was initially established in Dr Amor's laboratory. For patient 5, the parents did not allow a skin biopsy to be performed. The relative quantification of FOXG1 transcripts was performed on total mRNAs extracted from cultured fibroblasts (patients 3,6, 7, and 8) or from whole saliva (patient 5) (Figure 3a). In patients 3 and 7 , the constitutional deletions in the vicinity of FOXG1 are associated with a higher level of FOXG1 mRNAs (increased level 28-fold and 2-fold, respectively). In cells from whole saliva, the FOXG1 mRNAs level is also increased (twofold) in patient 5 who is a mosaic for the $14 \mathrm{q} 12$ deletion. Conversely, patient 8 with a $4.5 \mathrm{Mb}$ duplication including FOXG1 shows a 10 -fold decreased of mRNAs level. The modifications at the RNA level were confirmed at the protein level (Figure 3b). The increase at protein level is also much higher for patient 3 $(10 \times)$ as compared with patient $7(2 \times)$. These results are in favour of a cis-acting silencer in the deletions/duplication overlapping region in $14 \mathrm{q} 12$ that impacts FOXG1 expression. Surprisingly, the RNA/protein levels in patient 3 are much higher than in patients 5 and 7. There are several possible explanations for these variations. The FOXG1 expression quantification has been performed on fibroblast cell lines at different passages that were established from patients at different age. The genetic background is different from one patient to another. More importantly, the deletion sizes are different in patient 3 $(2.1 \mathrm{Mb})$, patient $5(0.4 \mathrm{Mb})$, and patient $7(0.62 \mathrm{Mb})$. The large deletion in patient 3 might remove additional sequences which coregulate the FOXG1 expression. Moreover, the FOXG1 gene is located in different local DNA sequence environments on the three deleted chromosomes. The chromatin conformation changes near the FOXG1 locus could also explain the higher FOXG1 expression in patient 3 .

\section{Physical mapping of long-range regulatory elements of FOXG1} We characterised three deletions in 14q12 that do not include FOXG1 in patients with clinical conditions suggestive of FOXG1-associated phenotypes (Figure 1a, patients 3, 5, and 7). The minimal deleted area extends from the proximal boundary in patient 5 (28945 423) to the distal boundary in patient 7 (29372 834). Putative long-range regulatory elements might be located in this $0.43 \mathrm{Mb}$ DNA segment located $600 \mathrm{~kb}$ distal to the FOXG1 coding sequence. We compared the $430-\mathrm{kb}$ human critical region with five other species by using two sequence alignment tools (ESPERR computational method and the
ECR browser) (Figure 1b). Five highly conserved non-coding elements (HCNE) were identified in the intergenic region between FOXG1 and PRKD1. These HCNEs were tested for gene enhancer activity in vivo in transgenic mice, these data are available by using the Vista Enhancer browser (http://enhancer.lbl.gov/). One out of five HCNEs act as an enhancer in the mouse forebrain, it was not considered as a candidate FOXG1-regulatory element. Indeed, according to our RT qPCR results the putative cis-regulatory sequence represses FOXG1.

\section{DISCUSSION}

We report on the clinical and molecular characterisation of seven new patients (four females and three males) with severe encephalopathies resulting from genetic abnormalities affecting FOXG1 at the genomic or the transcriptional levels. Two frameshift mutations correspond to a 1 bp deletion or duplication in a poly-C stretch (c.250_256) in FOXG1. Another recurrent duplication (c.460dupG) was previously reported in three patients in a poly-G mononucleotide repeat. ${ }^{5-6,17}$ The same c.256dupC frameshift has already been reported twice in patients with congenital RTT. ${ }^{7,18}$ The c.256del/dup is the second mutational hot spot affecting a mononucleotide repeat in FOXG1. We also describe five new CNVs in 14q12 in two males and three females. In two cases, the deleted area encompasses FOXG1. Interestingly enough, three deletions close but distal to FOXG1 involve all or part of PRKD1. In patient 7, the deletion affects only PRKD1.

Including this study, 26 point mutations, $25 \mathrm{CNVs}$, and $2 \mathrm{t}(2 ; 14)$ balanced translocations have been reported in patients with a FOXG1related phenotype. ${ }^{1-14,17-22}$ The male-to-female ratios is $7 / 26(27 \%)$ for point mutations and 13/25 (52\%) for CNVs. The preponderance of females in patients positive for a FOXG1 point mutation is most probably due to the initial description of FOXG1 deleterious alleles in females with congenital RTTs. ${ }^{2,4}$ CNVs in $14 \mathrm{q} 12$ consist of deletions $(14 / 25,56 \%)$ and duplications $(11 / 25,44 \%)$. The vast majority of FOXG1 duplications were found in males $(9 / 11,82 \%),{ }^{10-13}$ and are frequently associated with West syndrome $(8 / 11,72 \%)^{10-13}$ or epilepsy with mental retardation and severe speech impairment. ${ }^{11}$ Microcephaly is only present in 1 out of 11 patients with complete duplications of the FOXG1 gene. However, the role of duplication of FOXG1 in the pathogenesis of cognitive impairment and epilepsy is still a matter of debate. ${ }^{23,24}$

Combined with previous studies, our cohort suggests that the clinical phenotype associated with deleterious alleles affecting directly or indirectly FOXG1 is heterogeneous (Table $1,{ }^{2-6}$ ). In most articles, the FOXG1-related phenotype was reported as the congenital variant of RTT (MIM\#613454). Recently, the FOXG1-related encephalopathy was described as a clinically recognisable phenotype designated as the FOXG1 syndrome. ${ }^{6}$ According to this study, the core syndrome phenotype consists of severe postnatal microcephaly, severe mental retardation, absent language, dyskinesia, and corpus callosum hypogenesis. ${ }^{6}$ In addition, strabismus, protruding tongue, feeding difficulties, bruxism, and inexplicable laughing are often reported in patients heterozygous for a FOXG1-mutated allele. In three patients (2, 5, and 6), the phenotypes do correspond to the FOXG1 syndrome with severe postnatal microcephaly ( $-4 \mathrm{SD}$ to $-5.5 \mathrm{SD}$ ), severe mental retardation without any language development, dyskinesia, and abnormal brain development. Patient 7 presented with a clinical presentation compatible with the FOXG1 syndrome without corpus callosum dysgenesis. We, and others, previously described two females with a classical Rett linked to FOXG1 ${ }^{2,5}$ Again in this series, patient 1 heterozygous for the c.256dupC has a phenotype very reminiscent of RTT with a normal psychomotor development for the first 


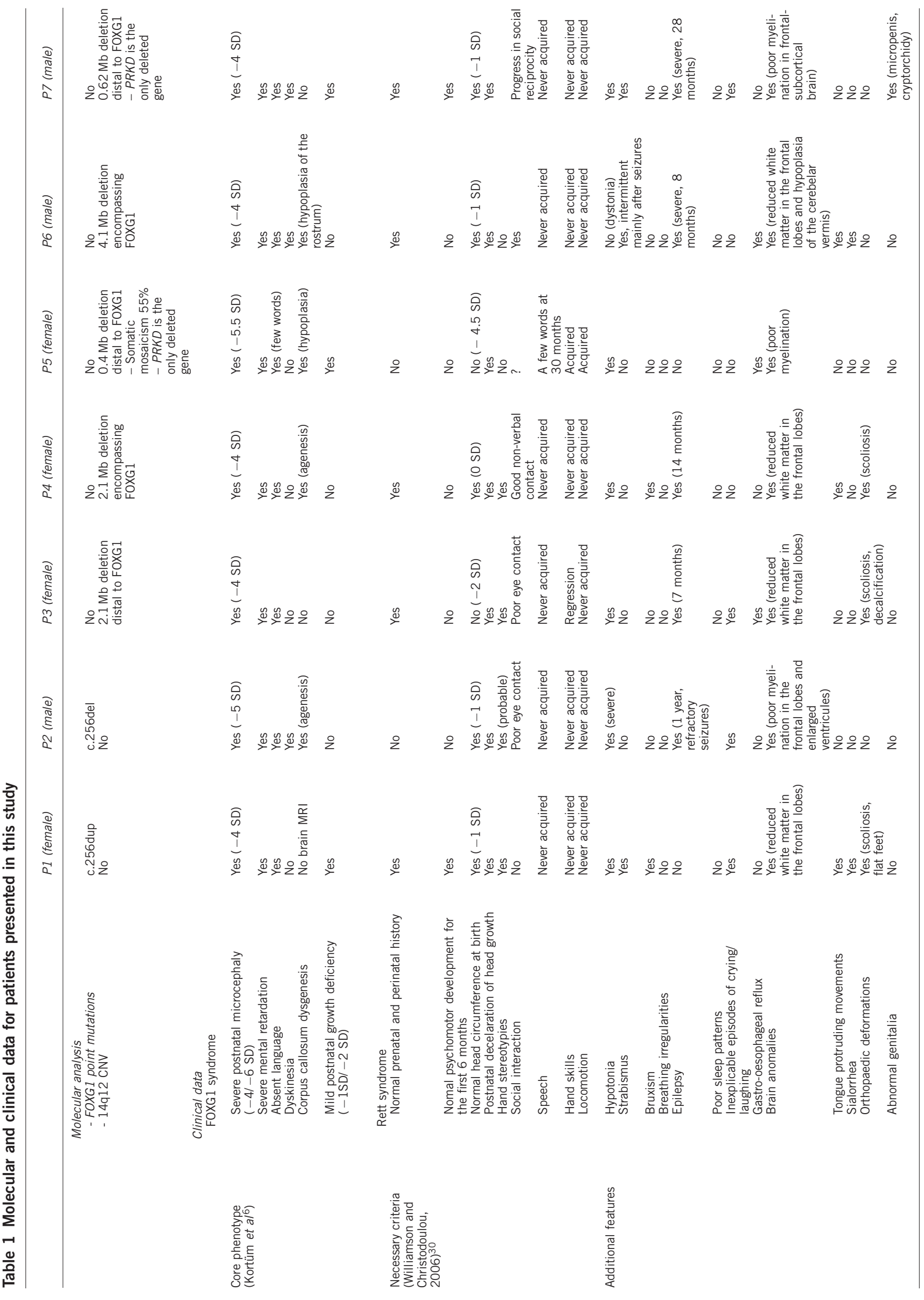



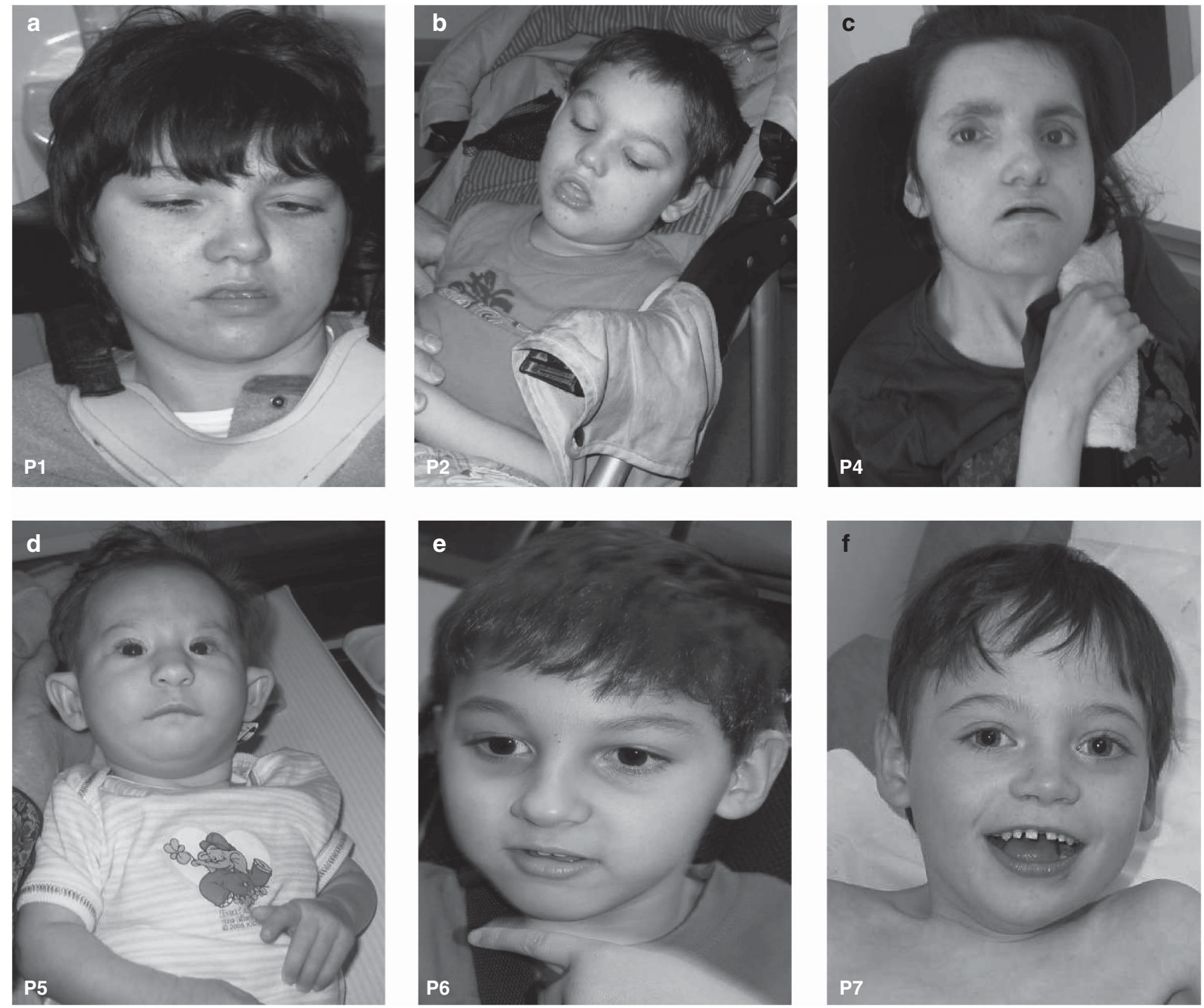

Figure 2 Clinical pictures of six out of seven patients presented in this study. Pictures (front view) of patients P1 (a), P2 (b), P4 (c), P5 (d), P6 (e), and P7 (f). No common facial feature is remarkable. Notice that patients are shown at very different ages.

6 months of life; however, she presented with a more severe acquired microcephaly than classically observed in RTT. One group hypothesised that deletion in the vicinity of FOXG1 may be associated with normal corpus callosum. ${ }^{6}$ Patients 3 and 7 presented with a normal corpus callosum. However, both patients 5 and DECIPHER 3810 with $14 \mathrm{q} 12$ deletions restricted to PRKD1 showed hypoplasia of the colossal commissure. Additional genetic and/or environmental factors probably explain different phenotypic outcomes in patients with very similar FOXG1 mutations.

Patients 3, 5, and 7 presented with small 14q12 deletions close but distal to FOXG1 (Figure 1a). Patient 5 is a somatic mosaic, only half of her lymphocytes are heterozygous for the 14q12 deletion. All three patients have a phenotype reminiscent to that of FOXG1mutated patients. The phenotype in patient DECIPHER 3810 with a deletion in the vicinity of FOXG1 (http://decipher.sanger.ac.uk/) (Figure 1a) overlaps somehow with that of FOXG1-mutated patients. Notably, the PRKD1 gene always lies within the deleted segment in all patients with a FOXG1-like phenotype associated with deletions distal to FOXG1 (this study, DECIPHER $3810,{ }^{6,8}$ ). In addition, two cases of $\mathrm{t}(2 ; 14)$ balanced translocations with a $14 \mathrm{q} 12$ breakpoint distal but close to FOXG1 have been reported in girls with phenotypes overlapping RTT with severe mental retardation and microcephaly ${ }^{6,14}$ (Figure 1a). PRKD1 is a serine/threonine kinase that regulates a variety of cellular functions. Protein kinase D1 stimulates DNA synthesis and cell proliferation, and phosphorylates histone deacetylase (HDAC) 5 that is involved in control of chromatin structure and gene expression. ${ }^{25,26}$ Protein kinase D1 also controls dendritic arborisation in hippocampal neurons. ${ }^{27}$ The PRKD1 kinase phosphorylates class II HDACs. Phosphorylation-dependant nuclear export of HDAC4 and HDAC5 results in de-repression of downstream target genes. ${ }^{25,28}$ Reduction of the PRKD1 kinase activity could explain the de-repression of FOXG1 transcription in patients 3, 5, and 7 whereas an increased kinase activity could downregulate FOXG1 expression in patient 8. To date, no clinical 
a

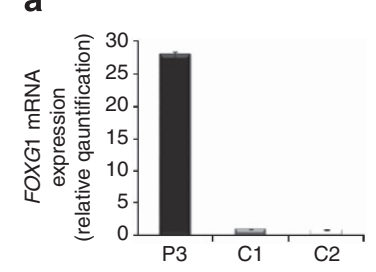

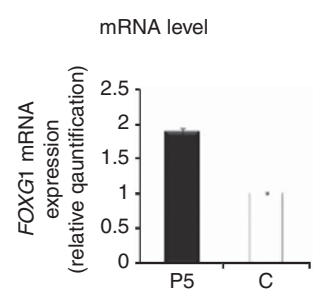

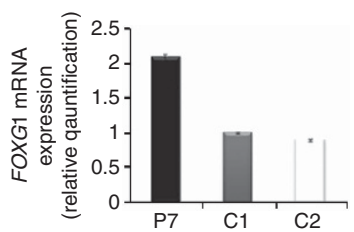

b

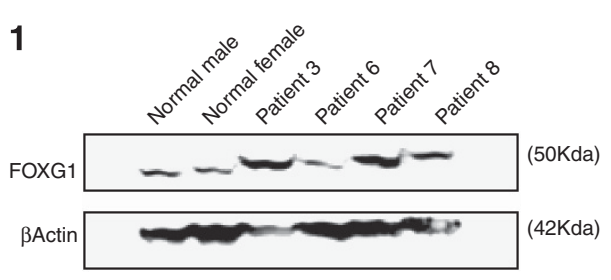

2

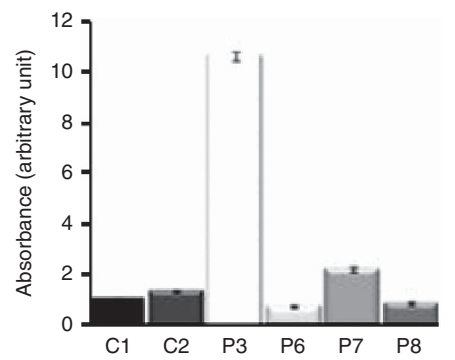

Figure 3 Quantification of the expression of FOXG1 at the mRNA and protein level. (a) Quantification of FOXG1 expression by RT-qPCR on total RNAs extracted from fibroblast cells (patients 3,6, 7, and 8) or from whole saliva (patient 5). FOXG1 mRNA level is increased in patients 3, 5, and 7 with a $14 \mathrm{q} 12$ deletion close to FOXG1. Conversely, the FOXG1 expression is decreased in patient 8 (duplication including FOXG1) and in patient 6 (deletion including FOXG1). qPCRs have been performed in triplicate. (b) Quantification of FOXG1 expression by western blot analysis on total proteins extracted from fibroblast cells (patients $3,6,7$, and 8). The RT-qPCR results were confirmed by the quantification of the endogenous FOXG1 protein in patients 3 , 6,7 , and 8 by immunoblotting with FOXG1 antibody. Densitometric quantification of the relative abundance of FOXG1 in each patient is presented in a bar graph. P: patient. C: control. $\beta$-actin was used as a standard in the quantification of the relative abundance of endogenous FOXG1.

entity has been associated with mutations or deletions in PRKD1. Although unlikely, mutations in PRKD1 could be responsible for a clinical condition overlapping the FOXG1/congenital RTT. More than a hundred FOXG1-negative patients with a severe encephalopathy were screened retrospectively by qPCR directed towards exons 2,10 , and 16 of PRKD1 (data not shown). For 11 patients with a clinical phenotype very evocative of the FOXG1-related encephalopathy, we also looked for point mutations in the coding sequence corresponding to the active site of the PRKD1 serine/threonine kinase. We did not find any large rearrangements or point mutation affecting PRKD1 in these patients.

The cis-acting regulatory element hypothesis could explain the Rett-like phenotype in patients heterozygous for a $14 \mathrm{q} 12$ microdeletion close to FOXG1. Our results provide additional support for the existence of telomeric sequences regulating the expression of FOXG1. 6,14 Three additional CNVs allow us to narrow down the locus for a putative regulatory element in a $0.42-\mathrm{Mb}$ DNA segment (28 945 423-29372 834, hg18) corresponding to the smallest region of overlap (Figure 1a). Expression studies on cultured fibroblasts demonstrated that the expression of FOXG1 is increased in patients 3,5 , and 7 and decreased in patient 8 with a microduplication encompassing the FOXG1 locus (Figure 3). A regulatory element located more than $0.6 \mathrm{Mb}$ away from the FOXG1 coding sequence seems to act as a silencer at the transcriptional level. This regulation needs to be confirmed in vivo during the forebrain development. An unexpectedly high number of HCNEs correspond to functional cis-regulatory regions that influence gene transcription. Disruption of non-coding DNA sequences with regulation function of developmental genes is an emergent mutational mechanism in genetic diseases. $^{29}$ Two HCNEs that were proposed as candidate long-range cis-regulatory elements for FOXG1 $1^{6}$ do not map within the critical region as defined in this study. We selected four candidate highly conserved elements (Figure 1b) without enhancer activity as assessed in transgenic mice. We sequenced these highly conserved regions in 11 patients with a clinical phenotype very evocative of a FOXG1-related encephalopathy and did not find any deleterious mutations (data not shown). Additional patients and further studies will be necessary to track and characterise FOXG1 cis-regulatory sequences. Interestingly enough, a $88 \mathrm{~kb}$ duplication at 14q12 encompassing FOXG1 and C14orf23 was found in a father and his son associated with a normal neurocognitive phenotype. ${ }^{23}$ This duplication in $14 \mathrm{q} 12$ does not include the putative cis-regulatory elements mapped in the present study. We believe that this additionnal duplication in $14 \mathrm{q} 12$ strengthens the FOXG1 long-range regulatory elements hypothesis. Importantly, the FOXG1 molecular screening strategy should include a quantitative approach to look for deletions in the critical region harbouring putative FOXG1 cis-acting regulatory elements as defined in this study. We perform in a routine practice qPCRs with amplicons located in PRKD1 (three amplicons) but also between FOXG1 and PRKD1 (four amplicons).

In conclusion, we expand the mutational spectrum in patients with FOXG1-related encephalopathies. We precise the core FOXG1 syndrome phenotype; 6 dysgenesis of the corpus callosum and dyskinesia are not always present in FOXG1-mutated patients. A severe microcephaly ( $-4 \mathrm{SD}$ to $-6 \mathrm{SD}$ ) is always present in FOXG1-positive patients. Three additional deletions in the vicinity of FOXG1 allow us to physically map a FOXG1-regulatory element in a $0.43-\mathrm{Mb}$ DNA segment encompassing the PRKD1 locus. In fibroblast cells, a cis-acting regulatory sequence located more than $0.6 \mathrm{Mb}$ away from FOXG1 acts as a silencer at the transcriptional level. These data are important for clinicians and for molecular biologists involved in the management of patients with severe encephalopathies compatible with a FOXG1-related phenotype.

\section{CONFLICT OF INTEREST}

The authors declare no conflict of interest. 


\section{ACKNOWLEDGEMENTS}

We gratefully acknowledge clinicians who have referred patients for MECP2/CDKL5/FOXG1 analyses, and more particularly Professor L OlivierFaivre, Dr F Giuliano, Dr S Julia, Dr V Layet, and Dr A Roubertie. We thank MD Devignes (LORIA, Vandoeuvre les Nancy, France) for help with bioinformatic analyses. This work was supported by grants (CPRC 04.9582, 2009) from the Centre Hospitalier Universitaire de Nancy and from the Association Française du Syndrome de Rett (AFSR). LA is funded by a grant MESR (Ministère de l'Enseignement Supérieur et de la Recherche).

1 Le Guen T, Fichou Y, Nectoux J et al: A missense mutation within the fork-head domain of the forkhead box G1 gene (FOXG1) affects its nuclear localization. Hum Mutat 2011; 32: E2026-E2035.

2 Philippe C, Amsallem D, Francannet $C$ et al: Phenotypic variability in Rett syndrome associated with FOXG1 mutations in females. J Med Genet 2010; 47: 59-65.

3 Mencarelli MA, Spanhol-Rosseto A, Artuso R et al: Novel FOXG1 mutations associated with the congenital variant of Rett syndrome. J Med Genet 2010; 47: 49-53.

4 Ariani F, Hayek G, Rondinella D et al: FOXG1 is responsible for the congenital variant of Rett syndrome. Am J Hum Genet 2008; 83: 89-93.

5 Van der Aa N, Van den Bergh M, Ponomarenko N, Verstraete L, Ceulemans B, Storm K: Analysis of FOXG1 is highly recommended in male and female patients with rett syndrome. Mol Syndromol 2011; 1: 290-293.

6 Kortum F, Das S, Flindt M et al: The core FOXG1 syndrome phenotype consists of postnatal microcephaly, severe mental retardation, absent language, dyskinesia, and corpus callosum hypogenesis. J Med Genet 2011; 47: 49-53.

7 Le Guen T, Bahi-Buisson N, Nectoux J et al: A FOXG1 mutation in a boy with congenital variant of Rett syndrome. Neurogenetics 2011; 12: 1-8.

8 Mencarelli MA, Kleefstra T, Katzaki $\mathrm{E}$ et al: 14q12 Microdeletion syndrome and congenital variant of Rett syndrome. Eur J Med Genet 2009; 52: 148-152.

9 Jacob FD, Ramaswamy V, Andersen J, Bolduc FV: Atypical Rett syndrome with selective FOXG1 deletion detected by comparative genomic hybridization: case report and review of literature. Eur J Hum Genet 2009; 17: 1577-1581.

10 Striano $P$, Paravidino R, Sicca F et al: West syndrome associated with $14 \mathrm{q} 12$ duplications harboring FOXG1. Neurology 2011; 76: 1600-1602.

11 Brunetti-Pierri N, Paciorkowski AR, Ciccone R et al: Duplications of FOXG1 in 14q12 are associated with developmental epilepsy, mental retardation, and severe speech impairment. Eur J Hum Genet 2011; 19: 102-107.

12 Yeung A, Bruno D, Scheffer IE et al: $4.45 \mathrm{Mb}$ microduplication in chromosome band 14q12 including FOXG1 in a girl with refractory epilepsy and intellectual impairment. Eur J Med Genet 2009; 52: 440-442.

13 Tohyama J, Yamamoto T, Hosoki $\mathrm{K}$ et al: West syndrome associated with mosaic duplication of FOXG1 in a patient with maternal uniparental disomy of chromosome 14. Am J Med Genet A 2011; 155A: 2584-2588.
14 Shoichet SA, Kunde SA, Viertel P et al: Haploinsufficiency of novel FOXG1B variants in a patient with severe mental retardation, brain malformations and microcephaly. Hum Genet 2005; 117: 536-544.

15 Quenard A, Yilmaz S, Fontaine $\mathrm{H}$ et al: Deleterious mutations in exon 1 of MECP2 in Rett syndrome. Eur J Med Genet 2006; 49: 313-322.

16 Bonnet C, Leheup B, Beri M, Philippe C, Gregoire MJ, Jonveaux P. Aberrant GRIA3 transcripts with multi-exon duplications in a family with $\mathrm{X}$-linked mental retardation. Am J Med Genet A 2009; 149A: 1280-1289.

17 Bahi-Buisson N, Nectoux J, Girard B et al: Revisiting the phenotype associated with FOXG1 mutations: two novel cases of congenital Rett variant. Neurogenetics 2010; 11: 241-249

18 Takahashi S, Matsumoto N, Okayama A et al: FOXG1 mutations in Japanese patients with the congenital variant of Rett syndrome. Clin Genet 2011; e-pub ahead of print 1 December; doi:10.1111/j.1399-0004.2011.01819.x.

19 Bisgaard AM, Kirchhoff M, Tumer Z et al: Additional chromosomal abnormalities in patients with a previously detected abnormal karyotype, mental retardation, and dysmorphic features. Am J Med Genet A 2006; 140: 2180-2187.

20 Papa FT, Mencarelli MA, Caselli R et al: A 3 Mb deletion in $14 q 12$ causes severe mental retardation, mild facial dysmorphisms and Rett-like features. Am J Med Genet A 2008; 146A: 1994-1998.

21 Roche-Martinez A, Gerotina E, Armstrong-Moron J, Sans-Capdevila O, Pineda M: [FOXG1, a new gene responsible for the congenital form of Rett syndrome.]. Rev Neurol 2011; 52: 597-602.

22 De Filippis R, Pancrazi L, Bjorgo $\mathrm{K}$ et al: Expanding the phenotype associated with FOXG1 mutations and in vivo FoxG1 chromatin-binding dynamics. Clin Genet 2011; e-pub ahead of print 17 November. doi:10.1111/j.1399-0004.2011.01810.x.

23 Amor DJ, Burgess T, Tan TY, Pertile MD: Questionable pathogenicity of FOXG1 duplication. Eur J Hum Genet 2012; 20: 595-596.

24 Brunetti-Pierri N, Cheung SW, Stankiewicz P: Reply to Amor et al. Eur J Hum Genet 2012; 20: 597.

25 Huynh QK, McKinsey TA: Protein kinase D directly phosphorylates histone deacetylase 5 via a random sequential kinetic mechanism. Arch Biochem Biophys 2006; 450: 141-148.

26 Kisfalvi K, Hurd C, Guha S, Rozengurt E: Induced overexpression of protein kinase D1 stimulates mitogenic signaling in human pancreatic carcinoma PANC-1 cells. J Cell Physiol 2010; 223: 309-316.

27 Czondor K, Ellwanger K, Fuchs YF et al: Protein kinase D controls the integrity of Golgi apparatus and the maintenance of dendritic arborization in hippocampal neurons. Mol Biol Cell 2009; 20: 2108-2120.

28 Calalb MB, McKinsey TA, Newkirk S, Huynh K, Sucharov CC, Bristow MR: Increased phosphorylation-dependent nuclear export of class II histone deacetylases in failing human heart. Clin Trans/ Sci 2009; 2: 325-332.

29 Benko S, Fantes JA, Amiel J et al: Highly conserved non-coding elements on either side of SOX9 associated with Pierre Robin sequence. Nat Genet 2009; 41: 359-364.

30 Williamson SL, Christodoulou J: Rett syndrome: new clinical and molecular insights. Eur J Hum Genet 2006; 14: 896-903.

Supplementary Information accompanies the paper on European Journal of Human Genetics website (http://www.nature.com/ejhg) 